\title{
Los Sistemas Participativos de Garantía en el fomento de los mercados locales de productos orgánicos
}

\author{
Sofía Boza Martínez \\ Facultad de Ciencias Agronómicas, Universidad de Chile, Santiago, Chile. \\ Email: sofiaboza@u.uchile.cl
}

\begin{abstract}
Resumen: La normativa sobre agricultura orgánica de los principales mercados está diseñada conforme al entendimiento de que la distancia entre el productor y el consumidor de alimentos orgánicos es considerable, lo cual no tiene que ser necesariamente cierto. En consecuencia se han desarrollado iniciativas orientadas a la utilización de canales comerciales cortos para la producción orgánica, basados asimismo en la participación de los agentes que los integran en los procesos de certificación. Dentro de este contexto destacan los Sistemas Participativos de Garantía como modelos de certificación participativa en red, los cuales buscan potenciar la generación de confianza y el aprendizaje mutuo. El presente artículo tiene como objetivo describir los principios en los cuales se fundamentan, así como el funcionamiento, de los Sistemas Participativos de Garantía acorde con una estrategia de fomento de la participación comunitaria, la agricultura tradicional y el desarrollo rural endógeno.
\end{abstract}

Palabras clave: agricultura orgánica, certificación, Sistemas Participativos de Garantía, América Latina

\section{Participatory Guarantee Systems in promoting local markets for organic products}

\begin{abstract}
The organic farming regulations in major markets are designed according to the understanding that the distance between producer and consumer of organic foods is considerable, which is not necessarily true. Consequently there have been developed initiatives to enhance the use of short sales channels for organic production, also based on the participation of the agents into the certification process. Within these initiatives, we highlight the Participatory Guarantee Systems as models of participatory network certification, which seek to promote trust and learning. This paper aims to describe the principles on which Participatory Guarantee Systems are based and their operation according with a strategy of enhancing community participation, traditional agriculture and endogenous rural development.
\end{abstract} Latin America

Key words: organic farming, certification, Participatory Guarantee Systems,

\section{Sistemas Participativos de Garantia para o desenvolvimento de mercados locais de produtos orgânicos}

Resumo: A regulamentação sobre os principais mercados de agricultura 
orgânica é projetado de acordo com o entendimento de que a distância entre produtor e consumidor de alimentos orgânicos é considerável, que não precisa ser necessariamente verdade. Conseqüentemente, eles têm desenvolvido iniciativas para o uso de canais de vendas curtas para a produção orgânica, também com base na participação dos agentes que integram o processo de certificação. Dentro deste contexto incluem Sistemas Participativos de Garantia como modelos de certificação de rede participativa, que buscam promover o desenvolvimento da confiança e aprendizado mútuo. Este trabalho tem como objetivo descrever os princípios em que se baseiam, ea operação de Sistemas Participativos de Garantia, de acordo com uma estratégia de promoção da participação da comunidade, a agricultura tradicional e endógena de desenvolvimento rural.

Palavras-chave: agricultura orgânica, certificação, Sistemas Participativos de Garantia, América Latina.

$$
* * *
$$

\section{Introducción}

Los continuos avances en el campo de la agronomía han contribuido a la obtención de mayores rendimientos por hectárea trabajada. Sin embargo, a este proceso ha ido aparejada en las últimas décadas una creciente preocupación por las consecuencias ambientales de la intensificación de la agricultura. Resultado inmediato de lo anterior es que en los años veinte del pasado siglo surge una primera corriente conducente a la constitución sistémica de la agricultura orgánica. A medida que este modelo novedoso se extendió, su trasfondo epistémico fue quedando en un segundo plano en favor de su concreción práctica en distintas escuelas.

A pesar de la variedad de tendencias en torno a la agricultura orgánica, se destacan dos lineamientos a este respecto. Por un lado tenemos el "modelo de sustitución de insumos", basado en una transición del uso de productos químicos de síntesis a enmiendas orgánicas. Esta visión se contrapone al "modelo agroecológico", según el cual la agricultura orgánica debe buscar la sustentabilidad ambiental de los cultivos, pero también la económica, social y cultural de los entornos donde se realizan. El primero de los enfoques señalados nace en las sociedades industrializadas con el objetivo de disminuir la degradación ambiental y de lograr una alimentación más saludable. Mientras tanto, la agroecología surge del campesinado de los países en desarrollo ampliando los objetivos anteriores (Guzmán, González \& Sevilla, 2000). Estos enfoques se complementan con dos visiones relativas a los sistemas de producción y comercialización orgánica: un "modo artesano” donde los canales de comercialización son cortos, por lo cual hay una relación de cercanía entre el consumidor y el productor; y el "modo neofordista" según el cual se produce a escala para el gran mercado (Buck, Getz \& Guthman, 1997). La agroecología trata de adaptarse de mejor manera al primero de los escenarios, buscando estrategias para su pervivencia. No obstante, la legislación sobre agricultura orgánica en los principales mercados de consumo responde al avance del segundo modelo. 
Por ende, las legislaciones mencionadas no están diseñadas para una estructura de canales comerciales cortos, sobre todo en la certificación de calidad de los productos. En consecuencia, se han desarrollado diversas experiencias considerando nuevos procesos de certificación, los cuales no estén basados en la labor de una entidad externa, sino en la fiscalización por parte de los propios agentes involucrados en la cadena de producción. Dentro de estas iniciativas destacan los Sistemas Participativos de Garantía (SPG), los cuales además de la certificación y etiquetado para las producciones orgánicas, buscan incentivar la construcción de redes de trabajo y aprendizaje mutuo vertebradoras de los territorios rurales donde se insertan.

El presente artículo tiene como objetivo describir los principios en los cuales se fundamentan, así como el funcionamiento, de los Sistemas Participativos de Garantía, acordes con una estrategia de fomento de los canales cortos de distribución, de la participación comunitaria y de la agricultura tradicional, consecuente con los principios de la agroecología. Dicha descripción se complementará con el estudio de tres SPG latinoamericanos: la Red Ecovida de Brasil, la Red de Agroecología de Uruguay y la Red Mexicana de Tianguis y Mercados Orgánicos.

\section{Los principios de la agroecología y su relación con el desarrollo endógeno}

El surgimiento de la agroecología está marcado por un proceso de recuperación por parte de la ciencia agronómica de las técnicas utilizadas tradicionalmente por los campesinos indígenas (principalmente latinoamericanos), que se desarrolla en las últimas décadas del siglo pasado. Este proceso, unido a una cada vez mayor concienciación hacia los conflictos ecológicos y sociales en el mundo rural, han conformado las pautas axiológicas en que se sustenta el movimiento agroecológico. En este contexto, la agroecología se basa en la identificación de la producción agrícola como un ecosistema, el cual debe ser gestionado en consonancia con la naturaleza de las relaciones que se dan en su interior. Como señala Hecht (1999) en la esencia misma del pensamiento agroecológico encontramos la visión del predio agrícola como un ecosistema más, donde se dan los mismos ciclos y procesos que en cualquier otra formación natural. La agroecología se enfocaría en el estudio de dichas relaciones ecosistémicas.

No obstante, no sólo serían los condicionantes de tipo biológico o medioambiental los que configurarían la estructura de los ecosistemas agrarios, de igual importancia resultan los factores sociales, económicos y culturales que envuelven a los agentes interconectados con ellos. Es por este motivo que la agroecología, a diferencia de otras escuelas del movimiento orgánico, le ha dado una gran importancia al estudio de la sociología agraria y del desarrollo rural. 
Esta visión más holística de los sistemas de producción (y comercialización) agrícola suele ir en consonancia con una opinión negativa de las consecuencias que la modernización de los mismos han tenido en devenir de las comunidades campesinas de los países en desarrollo. La principal acusación a este respecto es haber mermado la capacidad de los pequeños agricultores para proteger su modo de vida, en tanto en cuanto, se impone una agricultura de monocultivo de exportación. A su vez, a los agricultores se les plantea necesaria la adopción de nuevas técnicas e insumos procedentes en la mayor parte de los casos de países desarrollados.

Contrariamente, desde la perspectiva agroecológica, el desarrollo de los entornos rurales debe darse a partir de la puesta en valor de los recursos locales presentes en ellos. En palabras de Sevilla (2006: 205),

"Cada agroecosistema posee un potencial endógeno en términos de producción de materiales e información (conocimiento y códigos genéticos) que surge de la articulación histórica de cada trozo de naturaleza y de sociedad; es decir, de su coevolución (...) La Agroecología busca utilizar y desarrollar dicho potencial, en lugar de negarlo y remplazarlo por las estructuras y procesos industriales”.

Por tanto, la agroecología constituye una propuesta de desarrollo rural de origen endógeno, conforme la cual se ha concebido una estrategia de acción en los territorios rurales basada en la búsqueda de espacios que faciliten la generación de circuitos comerciales cortos, donde las agrupaciones de pequeños productores puedan ser protagónicas. Esta manera de entender el desarrollo rural ha llevado a que la agroecología, desde el punto de vista práctico, se haya decantado por la puesta en marcha de proyectos participativos en comunidades campesinas mediante, en muchos casos, la labor de organizaciones no gubernamentales.

No obstante, el pensamiento agroecológico ha tenido poca aceptación dentro de las legislaciones sobre producción orgánica vegetal y animal de los principales mercados de consumo de dichos alimentos (Estados Unidos, Unión Europea y Japón). El concepto de la agricultura orgánica que inspira dichas legislaciones pone el énfasis casi exclusivamente en la sustentabilidad medioambiental de la práctica agrícola orgánica. En contraste, la agroecología defiende una visión multidimensional de la sustentabilidad, donde el agricultor orgánico tenga que tomar en consideración las repercusiones socioeconómicas (e incluso culturales) de su actividad.

A continuación vamos a arrojar mayor luz con respecto a la contraposición de enfoques planteada, en base al análisis de las motivaciones y dificultades de los agentes protagónicos del cambio hacia sistemas productivos más sustentables: los agricultores. 


\section{El proceso de conversión de los agricultores a la producción orgánica}

La dificultad de identificar unificadamente el movimiento agrario orgánico viene a su vez de la variedad de motivaciones que le subyacen, principalmente una vez se extiende el consumo de su producción a un público cada vez mayor. Esto ha ocasionado que se desarrollen numerosas investigaciones para clarificar cuáles son las motivaciones que llevan a los productores orgánicos a su conversión. Los factores económicos, así como las preocupaciones sociales y morales, han sido destacadas repetidamente en la literatura (Mzoughi, 2011). Por ende, como recoge Armesto (2007) se insinúa la coexistencia de una agricultura orgánica enfocada a la obtención de unos resultados (en términos de rentabilidad) donde, en consecuencia, su principal razón de ser es la económica; y una agricultura orgánica que no incide tanto en el resultado debido a que tiene otras motivaciones, como el medioambiente, la salud o la ideología.

En lo que se refiere a los factores económicos, éstos no se basarían solamente en el balance entre los ingresos y costos privados, sino que las ayudas que el sector público destinase a la agricultura orgánica deben ser consideradas también como un claro incentivo a la conversión (Pietola \& Oude, 2001). Sin embargo, incluso la adscripción a los programas de ayudas dependería de características intrínsecas del productor como su situación económica, socio-demográfica, acceso a información y percepción de riesgo ambiental (Toma \& Mathijs, 2007).

Además de las motivaciones que conducen a la conversión a la agricultura orgánica, las dificultades que actúan como barreras para dicho proceso han sido ampliamente revisadas. En este sentido, Guzmán y Alonso (2007) las enumeran para el caso europeo dentro de cuatro categorías: técnicas, sociales, legales y económicas o de mercado. Las primeras se centran en la falta de referencias y conocimientos necesarios de los agricultores. Por su parte, las barreras sociales se refieren a la carencia de apoyos en el entorno del agricultor y la ausencia de asociacionismo en el sector, e incluso de mano de obra suficiente, para poner en marcha la conversión. Desde el punto de vista legal, los autores destacan como barreras: la desprotección ante la que se encuentra el agricultor frente a algunas fuentes de contaminación; la dificultad de registrar variedades tradicionales y comercializar semillas; y las distorsiones en los mercados que introduce la Política Agraria Común. Finalmente, las barreras económicas o de mercado se relacionan fundamentalmente con la ausencia de un entramado interno comercial fuerte.

Asimismo, en lo que tiene que ver con los procesos de certificación de calidad de la producción, la cuantía de los costos relacionados, así como la burocracia del proceso, actuarían como potenciales barreras para los pequeños agricultores a la hora de convertirse a la agricultura orgánica. A esto se suma la pérdida de soberanía del agricultor frente a su producción, 
teniendo que atender a normas que en casi todos los casos proceden de instancias lejanas a él.

Comprenderemos mejor la naturaleza de esta problemática analizando las tendencias actuales del mercado de los productos de alimentación orgánica. En 1991, más del 90\% de las ventas de orgánicos a nivel mundial se realizaba en pequeños mercados, tiendas especializadas o directamente en las fincas. Sin embargo, desde hace años la producción agrícola orgánica certificada se comercializa mayoritariamente en grandes superficies (Echeverría, 2007). A esto se suma que el destino de la producción orgánica certificada de la mayor parte de los países en desarrollo son los mercados de los países desarrollados del hemisferio norte. En este sentido, FiBL, IFOAM y SÖL (2012) señalan que el 49\% de los ingresos procedentes de la venta de productos de agricultura orgánica proviene del mercado de Estados Unidos, mientras que el 47\% se genera en los mercados de los distintos países dentro de Europa.

La tendencia resultante sería, por tanto, la configuración de un modelo de agricultura orgánica globalizado, donde "los espacios y las relaciones socioeconómicas locales están supeditadas a escalas lejanas de ámbitos de acción y control” (Cuéllar \& Torremocha, 2008).

La manera como se están diseñando las legislaciones nacionales en torno a la agricultura orgánica no ayuda en muchos casos a revertir esta situación, sino más bien todo lo contrario. Este hecho se hace especialmente notable si nos referimos a la producción orgánica en aquellos países que tengan como principales mercados de destino Estados Unidos y la Unión Europea (lo cual es mayoritario como hemos visto). Dadas las normas sobre importaciones agrícolas orgánicas que rigen ambos mercados, según las cuales se permite la entrada sólo de aquellos productos que hayan sido obtenidos atendiendo a criterios asimilables a los estándares impuestos en ellos, se están desarrollando en consonancia legislaciones en los países exportadores lo más parecidas posibles a las de los países de destino de su producción. Esta forma de regular potenciaría el conflicto en torno a la certificación por tercera parte dada su dependencia de disposiciones que son externas a la realidad del territorio.

A esto se suma que, exista o no regulación nacional, para poder cumplir con las normas de importación de los mercados agrícolas orgánicos más importantes, en algunos países la labor certificadora viene desarrollada fundamentalmente por entidades privadas extranjeras de reconocido prestigio a nivel internacional. Esto representa un fuerte sobrecosto para los productores de los países exportadores (Gómez, Gómez \& Schwentesius, 2000).

Es por todo ello que surge la inquietud de desarrollar sistemas de certificación alternativos que estén basados en un control efectuado principalmente por los agentes implicados de manera directa en el proceso productivo. En este contexto, los Sistemas Participativos de Garantía suponen 
una propuesta especialmente interesante en la consecución, más allá de la propia certificación, de una red de colaboración que contribuya a la configuración del territorio.

\section{Los Sistemas Participativos de Garantía (SPG)}

En la práctica se han dado distintos tipos de sistemas alternativos de certificación, cuya diferenciación depende esencialmente de qué relación con la producción tenga quien asuma la responsabilidad de verificar que ésta ha sido llevada a cabo siguiendo criterios de sustentabilidad. Así se tendrían: los sistemas de evaluación de primera parte, en los que son los propios agricultores los que garantizan la adecuación de sus productos; los sistemas de segunda parte, en los que la certificación la da el comercializador; y la evaluación por tercera parte, en la cual el control sería llevado a cabo por parte de una asociación de agricultores.

Conjuntamente a estos sistemas, tratando de tomar los puntos fuertes de cada uno de ellos, se han venido desarrollando los Sistemas Participativos de Garantía, dentro de un marco de certificación participativa en red. Mediante los SPG se pretende conseguir que sean los propios productores, y otros agentes implicados, los que verifiquen la adecuación de los alimentos para ser considerados como de agricultura orgánica. Como señala Cuéllar (2007) los SPG se basan en la idea de que quien mejor puede trabajar en contacto constante con el agricultor orgánico son otros agricultores cercanos, así como los consumidores de sus productos, organizando a los agentes mencionados en una red donde se desempeñen de manera conjunta.

Este procedimiento es especialmente coherente si el contacto entre los agentes es muy directo, es decir, si los circuitos comerciales son cortos. En este sentido, según los define IFOAM (2007) los SPG son específicos de comunidades individuales, de áreas geográficas, de ambientes culturales y de mercados. Asimismo, implican menos administración y costos más bajos que la certificación por tercera parte enfocada a la exportación.

\section{Principios básicos de los SPG}

En países de todo el mundo, tanto del Norte como del Sur, se han desarrollado numerosas experiencias relacionadas con los Sistemas Participativos de Garantía en la agricultura orgánica (Gómez, 2007). Pese a esta diversidad, Meirelles (2007) propone algunos principios básicos comunes a las iniciativas mencionadas:

- Visión compartida. Tanto los agricultores como los consumidores del Sistema tienen que entender los principios de gestión del mismo de forma común. 
- Participación. Se trata de impulsar que todos los agentes que estén interesados en los productos se impliquen a su vez en el Sistema.

- Transparencia. Los agentes implicados en el SPG deben tener la mayor cantidad de información posible a su disposición sobre el desarrollo del mismo.

- Confianza. Es el principio fundamental para garantizar el funcionamiento de los SPG. Los agentes interesados deben creer en la veracidad de lo certificado a través del Sistema para prolongar su implicación con el mismo.

- Proceso pedagógico. Se hace especial énfasis en la formación de los productores y en el fortalecimiento de sus lazos, lo cual les permita que su acción conjunta se siga desarrollando a largo plazo.

- Horizontalidad. No hay jerarquías de control, es decir, la decisión de aquello que se puede verificar como agricultura orgánica no queda en manos de ningún ente o grupo de personas, sino que todos los agentes del Sistema pueden intervenir en igual grado.

Estos principios buscan conseguir un aprovechamiento máximo de las características del territorio en el que se implanta el SPG, que sirva como motor del desarrollo social y económico del mismo, pero siempre promoviendo su mantenimiento y perpetuación.

\section{Proceso de funcionamiento de los SPG}

Los Sistemas Participativos de Garantía basan su control en una serie de normas y procedimientos establecidos. Puede darse el caso de que dichas normas técnicas se vinculen al cumplimiento del reglamento con vigencia legal en el lugar donde se implanta el Sistema Participativo, o bien a los estándares sobre agricultura orgánica de algún organismo internacional, o por último sean desarrollados por y para el propio SPG.

Una vez clarificado cuál será el marco normativo del SPG, se procede a la declaración de conformidad con la observación y seguimiento de éste en su producción por parte de cada agricultor que se implique como miembro del Sistema. Se conforman a su vez una serie de grupos de control, compuestos por agricultores vinculados que verificarán el cumplimiento de las normas por parte de los miembros del SPG. Para poder garantizar dicho cumplimiento, se conducen una serie de visitas periódicas cruzadas a las fincas. Estas dos etapas son fundamentales e imprescindibles en cualquier Sistema Participativo de Garantía.

En muchos casos, los SPG complementan lo anterior con verificaciones llevadas a cabo por agentes externos al entramado productivo del Sistema, como pueden ser agricultores pertenecientes a otras zonas, consu- 
midores o técnicos. Aunque no son etapas consustanciales al Sistema Participativo, sí que resultan deseables puesto que refuerzan la credibilidad del mismo e implican a un mayor número de agentes en el proceso de aprendizaje del SPG.

A continuación, con el objeto de ejemplificar lo explicado hasta ahora, se van a describir tres Sistemas Participativos de Garantía vigentes en América Latina.

\section{La implementación de los SPG en América Latina: algunas experiencias}

\section{Red de Agroecología Ecovida}

La Red de Agroecología Ecovida surge a finales de los años noventa en Brasil. Su objetivo es amparar a los pequeños y medianos agricultores orgánicos brasileños que no tienen los medios, o no se muestran conformes, con tener que acceder a entidades privadas para certificar sus productos, sobre todo cuando su venta pretende realizarse en el entorno próximo. Además de ello trata de fomentar el proceso de aprendizaje mutuo y la concienciación ambiental de los agentes implicados. En este sentido, en uno de sus documentos institucionales la Red de Agroecología Ecovida (2004) manifiesta que, de hecho, la certificación debe nacer de un proceso de generación de conciencia con la naturaleza de cada productor, así como con su necesidad de trabajar de manera cooperativa para generar las sinergias apropiadas.

Actualmente, la Red está conformada por 3.000 familias productoras que se organizan en más de 300 asociaciones y cooperativas distintas; además se cuenta con el apoyo técnico de más de tres decenas de ONGs y alrededor de 10 cooperativas de consumidores (Meirelles, 2010). Las asociaciones de agricultores son de pequeño tamaño, suelen contar con entre 5 y 10 miembros y deciden la manera en la que quieren realizar su sistema de encuentros y visitas. En un segundo nivel, por zonas geográficas, se configura lo que en la Red se denominan los Núcleos Regionales, los cuales están compuestos por varias asociaciones de productores, alguna ONG de asistencia técnica y algún grupo de consumidores, los cuales se reúnen de manera periódica y también establecen una metodología propia para realizar las visitas. A partir del Núcleo se conforma un Consejo de Ética, que será el que analice la información disponible sobre los agricultores para decidir si otorgarles o no el sello de la Red (Cuéllar, 2008).

Una vez analizada la organización de la Red Ecovida, señalar que los pasos concretos que los productores tienen que llevar a cabo dentro de ella para poder obtener el sello certificador son los siguientes: a) integración en la Red, b) solicitud de certificación al Núcleo Regional, c) cumplimentación del formulario para la certificación, d) envío del formu- 
lario al Consejo de Ética, e) análisis del formulario por el Consejo y visita al predio, f) toma de decisión por parte del Consejo y g) aprobación de la certificación por el Núcleo Regional. A pesar de la importante exigencia que supone poner el proceso mencionado en marcha, se calcula que el costo anual por productor dentro de la Red es de entre 15 US\$ y 20US\$ (Meirelles, 2010).

Las normas por las que se guía en su proceso certificador la Red Ecovida como Sistema Participativo de Garantía han sido elaboradas a través del trabajo de varias organizaciones del campo de la agricultura orgánica, motivadas por la colaboración del Ministerio de Agricultura brasileño y por las ideas vertidas por los participantes en los distintos encuentros de la Red. Cabe destacar que en dicha reglamentación se da gran importancia, además de a los principios técnicos de producción, a la observancia de los derechos laborales, a la promoción de la soberanía alimentaria y a la participación igualitaria de todos los miembros de la Red.

En este sentido, señalar por último que el soporte de la legislación brasileña sobre agricultura orgánica ha sido muy importante para lograr que la Red Ecovida alcanzara una extensión tan amplia. Un buen ejemplo lo encontramos en el contenido de la Ley $\mathrm{n}^{\circ} 10.831$ sobre la actividad agropecuaria orgánica en Brasil (reglamentada a través del Decreto nº 6323 , de diciembre de 2007). Dentro de dicha norma se señala que aquellos productores familiares que se dediquen a la venta directa al interior de un sistema social establecido podrán prescindir de la certificación oficial, siendo ésta de carácter meramente facultativo, siempre que se garantice la transparencia en la producción y haya mediado una inscripción en el órgano fiscalizador. De hecho, desde 2010 la Asociación Ecovida es reconocida por el Ministerio de Agricultura de Brasil como "Organismo Participativo de Garantía de Conformidad” lo que la valida de manera oficial como entidad que puede certificar la calidad orgánica de las producciones agrícolas.

\section{Red de Agroecología de Uruguay}

Como en otros países de América Latina, realizar el proceso de conversión hacia la agricultura orgánica fue visto en Uruguay como una oportunidad de aumentar y diversificar las exportaciones a los mercados del hemisferio norte. La necesidad de generar garantías suficientes en los canales comerciales internacionales para la introducción de los productos orgánicos uruguayos, llevó a que a mediados de los años noventa se creara la Asociación Certificadora de la Agricultura Ecológica como entidad de control, cuyo aval era el sello URUCERT.

Sin embargo, aquellos agricultores que realizaban sus cultivos siguiendo un sistema orgánico, pero que estaban interesados en comercializarlos en su entorno próximo, no consideraron necesario el someterse a controles para que sus productos exhibieran el sello URUCERT. A esto se sumaba que, para vender mediante canales cortos, no se hacía valer la exigencia legal de que los productos de agricultura orgánica estuvieran for- 
malmente certificados. En consecuencia, en Uruguay para la agricultura orgánica “con excepción de las cadenas de supermercados, los canales de comercialización no eran muy exigentes en cuanto al uso de sellos, y tampoco existían controles estatales sobre los productos comercializados” (Gómez, 2007:12).

Esta doble sistematización del mercado de la agricultura orgánica uruguayo, llevó a que se entendiera como necesaria la complementación del sistema de certificación existente para tratar de atender también a los productores que distribuían sus mercaderías en canales cortos. Así, en 2005 surge la Red de Agroecología de Uruguay como iniciativa de la Asociación de Productores Orgánicos de Uruguay, tratando de integrar a productores, consumidores, organizaciones no gubernamentales, instituciones gubernamentales y centros de investigación, implicados en alguna medida en el sector de la agricultura orgánica en el país.

Desde sus primeros años de funcionamiento, la Red de Agroecología de Uruguay ha desarrollado un programa de certificación participativa. En él la decisión de conceder o no la certificación a un productor se articula a través de los Consejos de Ética y Calidad que designa cada Regional en los cuales se divide la Red. Para asegurar la transparencia en el proceso, cualquier miembro de la Red puede acceder a la información sobre cómo éste ha sido llevado a cabo, además de que ningún integrante del Consejo de Ética y Calidad puede participar en los fallos sobre cultivos de productores con los que esté vinculado de alguna manera.

La certificación participativa puede ser solicitada a la Red por los agricultores tanto de manera individual como asociada. En cualquier caso los productores reciben los siguientes documentos: la declaración del agricultor, el manual operativo y la guía de formación, así como la solicitud y el plan de manejo anual. Si mediante el análisis de esta información el agricultor/es considera que está siguiendo las normas del programa de certificación de la Red completa la solicitud y el plan de manejo. El Regional otorga al grupo o individuo interesado un presupuesto de los costos de la certificación, si éste lo acepta y paga el cincuenta por ciento, recibirá posteriormente la vista del Consejo de Ética y Calidad del Regional en su finca, el cual decidirá si se aprueba la certificación y la consiguiente obtención del sello de la Red de Agroecología.

En este contexto, los principios que la Red de Agroecología de Uruguay manifiesta que le son propios y que trata de transmitir para guiar las acciones de sus organismos descentralizados son: la sustentabilidad, el cuidado de la biodiversidad y los recursos, la soberanía alimentaria, la participación, la solidaridad y el trabajo digno respetando los derechos humanos.

\section{Red Mexicana de Tianguis y Mercados Orgánicos}

En México la búsqueda de un mayor acercamiento de la producción orgánica nacional al consumidor doméstico tiene como ejemplo la labor de 
la Red Mexicana de Tianguis y Mercados Orgánicos. Dicha entidad, la cual comenzó a operar en 2004, se encuentra conformada en la actualidad por más de veinte tianguis o mercados locales de productos orgánicos emplazados en nueve estados del país (Baja California Sur, Chiapas, Estado de México, Jalisco, Morelos, Oaxaca, Puebla, Tlaxcala y Veracruz) y el Distrito Federal. A pesar de la popularidad que ha alcanzado la Red, los mercados que la componen han tenido que hacer frente a numerosos retos como son: la escasez de recursos materiales y humanos, las dificultades en la organización de programas de capacitación y la falta de abastecimiento suficiente de productos orgánicos obtenidos en el entorno local (Gómez, Gómez, Nelson \& Schwentesius, 2008).

No obstante, la Red Mexicana de Tianguis y Mercados Orgánicos ha participado también de numerosos logros a lo largo de sus años de funcionamiento. Uno de los más importantes es el desarrollo de un Sistema Participativo de Garantía para la certificación de las producciones de los agricultores miembros de sus mercados. A este respecto, la Red ha ejercido una determinante influencia para que la certificación orgánica participativa en la agricultura familiar y/o en las organizaciones de pequeños productores esté considerara en el artículo 24 de la Ley de Productos Orgánicos de febrero de 2006. Dicho reconocimiento se complementa con un convenio firmado en 2010 entre la Red y el Servicio Nacional de Sanidad, Inocuidad y Calidad Alimentaria de México donde se destaca la voluntad de promover de manera conjunta la certificación orgánica participativa para la producción a pequeña escala a partir de la puesta en marcha de un proyecto titulado "Procesos de Certificación Participativa”.

Asimismo, la Red Mexicana de Tianguis y Mercados Orgánicos acorde con una visión de que los SPG deben fomentar la generación de redes, incluye dentro de sus actuaciones visitas cruzadas entre agentes pertenecientes a los diferentes mercados y tianguis, reuniones y eventos conjuntos, jornadas de formación y publicación de documentos temáticos de interés. A su vez, se intenta ampliar la diversidad de actores locales relacionados con el sector de la producción orgánica que se impliquen en el funcionamiento de la Red y de su certificación.

Finalmente, señalar que la Red Mexicana de Tianguis promueve entre sus miembros, además del cumplimiento de estándares relacionados con la protección medioambiental, la observancia de ciertos criterios adicionales orientados al logro de una sustentabilidad socioeconómica. Ejemplo de ello es el fomento de un papel activo de las mujeres dentro de la organización, para así combatir la discriminación de género tan presente en los entornos rurales.

\section{Conclusiones}

Los sistemas de certificación alternativa para la agricultura orgánica se basan en que la verificación de los productos sea llevada a cabo por 
uno o más agentes implicados en la cadena productiva, comercial y/o de consumo de los mismos. Los Sistemas Participativos de Garantía (SPG) son un ejemplo concreto dentro de este escenario. Se distinguen principalmente porque en ellos es muy importante que el compromiso y el grado en el cual se involucran en el funcionamiento del sistema los agentes relacionados con la producción sea fuerte. Bajo esta premisa, desarrollar el control de los productos de agricultura orgánica no sería entendido como el objetivo principal de los SPG, sino que el propio proceso pedagógico, la generación de confianza y el establecimiento de un grupo sólido que actúe conjuntamente, serían metas prioritarias. En definitiva, no es la ausencia de una entidad certificadora externa lo que le da sentido e identidad a los SPG, sino el empoderamiento y la participación de los agentes del territorio, puesto que esto último es lo que genera conocimiento. Dicha visión de la agricultura orgánica es coincidente con los principios de la agroecología en su faceta de promoción de un desarrollo rural endógeno. Asimismo, es importante destacar que los SPG no están pensados para sustituir a los sistemas de certificación orgánica oficiales, sino para complementarlos en ciertos casos en pequeñas producciones agrícolas y canales comerciales cortos.

Por su parte, las iniciativas de implantación de Sistemas Participativos de Garantía en América Latina muestran una evolución en todo caso positiva, lo que haría pensar en la oportunidad de su réplica. En este sentido, las experiencias analizadas convergerían hacia una agricultura ideada como herramienta de preservación de la pervivencia de los espacios naturales, garantizando asimismo las posibilidades de la población autóctona de permanecer dignamente en su medio, conservando, potenciando y desarrollando saberes tradicionales muy valiosos. 


\section{Bibliografía}

Armesto, X. A. (2007), "El Concepto de la Agricultura Ecológica y su Idoneidad para Fomentar el Desarrollo Rural Sostenible”, en Boletín de la Asociación de Geógrafos Españoles, nº 43: 155-177.

Buck, D, Getz, C \& Guthman, J. (1997), “From Farm to Table: The Organic Vegetable Commodity Chain of Northern California”, en Sociologia Ruralis, vol. 37(1): 3-20.

Cuéllar, M. C. (2007), “Sistemas de Garantía Participativos. Socializando la credibilidad de los productores ecológicos”, en Revista FACPE, $\mathrm{n}^{\circ} 1$ : 16-17.

Ídem (2008), Hacia un sistema participativo de garantía para la producción ecológica en Andalucía. ISEC Universidad de Córdoba, Córdoba.

Ídem \& Torremocha, E. (2008), Proceso de construcción y regulación de un Sistema Participativo de Garantía para la producción ecológica en Andalucía. [Documento de trabajo]

Echeverría, F. (2007), “Organic Production and Trade: Contributions, opportunities, challenges and constraints", ponencia presentada en Environmental Requirements and Market Access: Turning challenges into opportunities. Naciones Unidas, Ginebra.

FiBL, IFOAM \& SÖL (2012), "The world of organic agriculture: statistics and emerging trends 2010”. Documento disponible en: <http:// www.organic-world.net/yearbook-2011.html>.

Gómez, A. (2007), “Certificación participativa: El caso de la Red de Agroecología en Uruguay”, en Leisa revista de agroecología, vol. 23(1): $10-13$.

Gómez, L., Gómez, M. Á., \& Schwentesius, R. (2000). Desafíos de la Agricultura Orgánica. Editorial Mundi-Prensa México, México D.F.

Gómez, M. Á., Gómez, L., Nelson, E. \& Schwentesius, R. (2008), “Un movimiento orgánico local que crece: la Red Mexicana de Mercados Orgánicos”, en LEISA revista de agroecología, vol. 24(1): 18-21.

Guzmán, G., González, M., \& Sevilla, E. (2000), Introducción a la agroecología como desarrollo rural sostenible. Ediciones Mundi-Prensa, Madrid.

Guzmán, G. \& Alonso, A. (2007), “La investigación participativa en agroecología: una herramienta para el desarrollo sustentable” en Ecosistemas, vol. 16 (1): 16-27. 
Hecht, S. B. (1999). “La evolución del pensamiento agroecológico”. En M. Á. Altieri, Agroecología: bases científicas para una agricultura sustentable (págs. 15-30). Editorial Nordan-Comunidad.

IFOAM (2007), “La Agricultura Ecológica y los Sistemas de Garantía Participativos. Comercialización y Apoyo para los Productores Ecológicos de Pequeña Escala”. Documento disponible en: <http://www.ifoam.org>

Meirelles, L. (2007), “Sistemas Participativos de Garantía: origen, definición y principios”, en Revista de Agricultura Ecológica de AGRECOL, n ${ }^{\circ}$ 7.

Meirelles, L. (2010). "Sistemas Participativos de Garantía (SPG) en Brasil”. En VV.AA., Sistemas de Garantía para productos orgánicos en mercados locales y nacionales. (págs. 19-30). Instituto Interamericano de Cooperación para la Agricultura, San José de Costa Rica.

Mzoughi, N. (2011), "Farmers' adoption of integrated crop protection and organic farming: Do moral and social concerns matter?” en Ecological Economics, vol. 70: 1536-1545.

Pietola, K. \& Oude, A. (2001), "Farmer response to policies promoting organic farming technologies in Finland", en European Review of Agricultural Economics, vol. 28: 1-15.

Red de Agroecología Ecovida. (2004). Cuaderno de Formaçao: Certificaçao Participativa de Produtos Ecológicos. Red de Agroecología Ecovida, Florianópolis.

Sevilla, E. (2006). De la sociología rural a la agroecología. Editorial Icaria, Barcelona.

Toma, L. \& Mathijs, E. (2007), “Environmental risk perception, environmental concern and propensity to participate in organic farming programmes”, en Journal of Environmental Management, vol. 83: 145157.

Recibido: 10.12.2012

Aceptado: 07.03.2013 